\title{
Socioeconomic inequalities in outcome of pregnancy and neonatal mortality associated with congenital anomalies: population based study
}

\author{
Lucy K Smith research fellow, Judith L S Budd EMSYCAR coordinator, David J Field professor of \\ neonatal medicine, Elizabeth S Draper professor of perinatal and paediatric epidemiology
}

Department of Health Sciences, University of Leicester, Leicester LE1 6TP, UK

\begin{abstract}
Objectives To investigate socioeconomic inequalities in outcome of pregnancy and neonatal mortality associated with congenital anomalies.

Design Retrospective population based registry study.

Setting East Midlands and South Yorkshire regions of England (representing about 10\% of births in England and Wales).

Participants All registered cases of nine selected congenital anomalies with poor prognostic outcome audited as part of the United Kingdom's fetal anomaly screening programme with an end of pregnancy date between 1 January 1998 and 31 December 2007.
\end{abstract}

Main outcome measures Socioeconomic variation in the risk of selected congenital anomalies; outcome of pregnancy; incidence of live birth and neonatal mortality over time. Deprivation measured with the index of multiple deprivation 2004 at super output area level.

Results There were 1579 fetuses registered with one of the nine selected congenital anomalies. There was no evidence of variation in the overall risk of these anomalies with deprivation (rate ratio for the most deprived 10th with the least deprived 10th: $1.05,95 \%$ confidence interval 0.89 to 1.23). The rate ratio varied with type of anomaly and maternal age (deprivation rate ratio adjusted for maternal age: 1.43 (1.17 to 1.74) for non-chromosomal anomalies; 0.85 (0.63 to 1.15) for chromosomal anomalies). Of the nine anomalies, $86 \%$ were detected in the antenatal period, and there was no evidence that this varied with deprivation (rate ratio $0.99,0.84$ to 1.17 ). The rate of termination after antenatal diagnosis of a congenital anomaly was lower in the most deprived areas compared with the least deprived areas (63\% $v 79 \%$; rate ratio $0.80,0.65$ to 0.97 ). Consequently there were significant socioeconomic inequalities in the rate of live birth and neonatal mortality associated with the presence of any of these nine anomalies. Compared with the least deprived areas, the most deprived areas had a $61 \%$ higher rate of live births $(1.61,1.21$ to 2.15$)$ and a $98 \%$ higher neonatal mortality rate $(1.98,1.20$ to 3.27$)$ associated with a congenital anomaly.

Conclusions Antenatal screening for congenital anomalies has reduced neonatal mortality through termination of pregnancy. Socioeconomic variation in decisions regarding termination of pregnancy after antenatal detection, however, has resulted in wide socioeconomic inequalities in liveborn infants with a congenital anomaly and subsequent neonatal mortality.

\section{Introduction}

Congenital anomalies are a major cause of neonatal and infant mortality, especially in developed countries, accounting for around $30 \%$ of infant deaths in the UK. ${ }^{1}$ Furthermore they explain a quarter of the widening socioeconomic inequality in neonatal mortality in England. ${ }^{2}$ Understanding how these inequalities relating to congenital anomalies arise is key to implementing effective public health interventions to reduce socioeconomic inequalities in infant and neonatal mortality.

Socioeconomic inequalities in congenital anomalies have been shown to exist in the rates of stillbirth and perinatal, neonatal, and infant mortality. ${ }^{1-4}$ Research has shown an increasing risk of non-chromosomal anomalies with increasing deprivation ${ }^{5}$ and, in contrast, a decreasing risk of chromosomal anomalies, though this latter finding is predominantly because of the increased risk of chromosomal anomalies with increasing maternal age. ${ }^{5}$ The influence of socioeconomic deprivation along the pathway from antenatal detection to delivery and possible neonatal mortality, however, is not fully understood because of the lack of rigorous data in the antenatal period. Countries that have introduced the use of prenatal diagnostic techniques and access to termination of pregnancy because of congenital anomaly have seen large reductions in neonatal mortality rates, ${ }^{6-9}$ unlike those countries with more restrictive policies on pregnancy termination. ${ }^{10}$ Nevertheless, the impact of these secondary preventative measures might vary with socioeconomic deprivation in terms of access to, and timing of, antenatal detection services through to the provision of information, the interpretation of risk, and the consequent decision regarding continuation or termination of a pregnancy.

Evidence is sparse. A systematic review of studies in the United Kingdom showed no evidence of social inequalities in the uptake of prenatal screening, ${ }^{11}$ while research in Northern Ireland, where access to termination services is much more restricted, showed 
inequalities in both the offer and uptake of screening. ${ }^{11}{ }^{12}$ Further research suggests that socioeconomic differentials in decision making after antenatal detection are because of differences in maternal age. ${ }^{13}$ The term "congenital anomaly" covers a wide spectrum from the relatively minor to those with an exceptionally poor prognostic outcome, and it is the latter that will be most affected by secondary preventative measures.

We used data from a large congenital anomaly register in England (covering about 10\% of the births in England and Wales) for 1998-2007 to investigate socioeconomic inequalities in the risk of congenital anomalies with a poor prognosis from antenatal diagnosis to end of pregnancy. We explored the impact of variations in rates of termination of pregnancy for congenital anomaly on rates of stillbirth, live birth, and neonatal mortality associated with congenital anomaly to aid understanding of the reasons for the widening socioeconomic inequalities in neonatal and infant mortality in England.

\section{Methods}

\section{Study population}

The East Midlands and South Yorkshire (England) congenital anomaly register (EMSYCAR) currently covers about 74000 births annually (about a 10th of all births in England and Wales). We excluded data for Northamptonshire, which joined the register in 2003, leaving a geographical area with about 60000 births annually. This register is population based and includes all congenital anomalies in fetuses and infants of mothers living within the region at the time of delivery. It includes live births, stillbirths (from 24 weeks of gestation), spontaneous fetal loss (before 24 weeks of gestation), or termination of pregnancy because of fetal anomaly at any gestational age. The register uses multiple sources of case ascertainment from within the care pathway, including antenatal ultrasonography, antenatal screening, delivery reports, birth notifications, pathology, cytogenetics, clinical genetics, and paediatric surgery. All reported anomalies are coded according to the 10th revision of the international classification of disease (ICD-10). Information on maternal age and ethnicity, mother's postcode of residence at delivery, end date of pregnancy, and gestation at delivery were available from data collected by the register. We included in our study fetuses with an anomaly with an end of pregnancy date between 1 January 1998 and 31 December 2007.

As the focus of our study was how socioeconomic inequalities arise along the pathway from antenatal detection to delivery, we selected serious congenital anomalies, which we defined as either incompatible with life or associated with severe morbidity but which also have screening systems in place for a precise antenatal diagnosis to allow parents to make an informed decision about continuation of the pregnancy. As a starting point, we therefore used the 11 anomalies identified by the fetal anomaly screening programme in the UK (see table 1). ${ }^{14} \mathrm{We}$ excluded two anomalies - cleft lip and gastroschisis-as they were much less likely to have a poor prognostic outcome. We also chose to include only those conditions where the ICD-10 code uniquely identified an anomaly for which there was a high level of certainty about an adverse prognosis, resulting in nine anomalies that met these criteria: two chromosomal anomalies (trisomy 13 and trisomy 18) and seven non-chromosomal anomalies (anencephaly, spina bifida, hypoplastic left heart, bilateral renal agenesis, lethal skeletal dysplasia, diaphragmatic hernia, and exomphalos). Where fetuses were registered with a chromosomal diagnosis, we considered any coexisting congenital anomalies as secondary to the underlying chromosomal problem, rather than separate non-chromosomal anomalies, as such associations are well established. Additional information on antenatal detection was obtained from the register on these selected anomalies, including the method and timing of diagnosis. An anomaly was deemed to be "antenatally detected" if the date of detection of the exact, or a closely related, anomaly predated delivery or the date of detection of an antenatal soft marker related to the anomaly present at delivery predated the date of delivery.

We obtained denominator data on live birth (from the Office for National Statistics) and stillbirth (from the Centre for Maternal and Child Enquiries (CMACE)) by year of birth, mother's age, and the 10th of deprivation of the mother's residence at the time of birth for the study period (1 January 1998 to 31 December 2007). Deprivation was measured with an area level measure, the index of multiple deprivation $2004 .^{15}$ This measure comprises seven domain indices at the super output area level, which relate to income deprivation, employment deprivation, health deprivation and disability, education, skills and training deprivation, barriers to housing and services, and living environment deprivation and crime. Super output areas are the smallest areas for which these deprivation data are available; although some degree of heterogeneity will exist within them, the small size of the areas (only 1500 residents) limits this. We ranked all super output areas in England by deprivation score and divided them into 10 groups with about equal populations of births: 1 (least deprived) to 10 (most deprived). The geographical area covered by this study has a slightly more deprived profile compared with England (see table 2). Ideally analyses would be based on mother's postcode at conception, but this was not available from the register. Analyses were therefore based on mother's postcode at delivery. To assess the potential impact of changes in mother's residence through the pregnancy on the observed socioeconomic inequalities we looked at deprivation at antenatal detection and delivery for those fetuses where this information was available - that is, fetuses detected in the antenatal period from pregnancies that did not end in termination.

Analyses were undertaken at the individual case (fetus or baby) level. Poisson regression models ${ }^{16}$ were used to assess trends in the six outcome measures by deprivation 10th over time (1998-2002; 2003-7):

- Rate of selected anomalies in utero (all cases of selected anomalies whether identified in the antenatal period or at birth) (denominator: total live births, still births, and known late fetal losses and terminations of pregnancy-that is, those registered with a fetal anomaly)

- Rate of antenatal detection (denominator: all cases of selected anomalies)

- Rate of termination of pregnancy because of fetal anomaly (denominator: antenatally detected cases)

- Rate of fetal loss or stillbirth with an anomaly (denominator: total live births, stillbirths, and known late fetal losses - that is, those registered with a fetal anomaly)

- Rate of live births with an anomaly (denominator: total live births)

- Rate of neonatal mortality of infants with an anomaly (denominator: total live births).

Data on terminated pregnancies and fetal losses before 24 weeks' gestation were available only when they were associated with a congenital anomaly. Only these terminations and late fetal losses were included in the denominators for calculation of rates of anomalies "in utero." Models were fitted for all anomalies combined and then separately for chromosomal and 
non-chromosomal anomalies. Interactions were explored to assess the change in the effect of deprivation over time. We then included maternal age $(<20,20-24,25-29,30-34,>35)$ in the models to assess its influence on any observed socioeconomic inequality. Confidence limits were obtained with the delta method. ${ }^{17}$ The numbers of live births and stillbirths were not available by ethnic group, though ethnic group was recorded as part of the register ( $86 \%$ complete) and so we could explore the confounding effect of ethnicity on rates of antenatal detection and termination of pregnancy. We included ethnicity (classified into four groups: white British, Asian or Asian British (Indian), Asian or Asian British (Pakistani), and other or missing) in the regression models to assess whether the inclusion of ethnic group attenuated any observed socioeconomic inequalities in the rate of antenatal detection and termination of pregnancy. The effect of gestational age at detection of anomaly on termination of pregnancy was also assessed $(<17$, 18-21, 22-23, >24 weeks' gestation).

We undertook sensitivity analyses using data on all registered congenital anomalies to assess whether patterns in outcome of pregnancy by deprivation for the nine selected anomalies were similar to those for all registered anomalies.

\section{Results}

From 1998 to 2007 there were 1712 separate registrations of the nine anomalies. We had full information on postcode and maternal age for 1695 (99\%). These 1695 registrations represented 1472 fetuses with one of the nine selected anomalies and 107 fetuses with two or more of the nine anomalies, making 1579 fetuses in total (table 1). Antenatal detection rates ranged from $97 \%$ for anencephaly to $77 \%$ for diaphragmatic hernia. Of those antenatally detected, over $80 \%$ of fetuses diagnosed with anencephaly, bilateral renal agenesis, trisomy 13 , or trisomy 18 ended in termination and less than $6 \%$ of fetuses with these anomalies were alive at birth and survived the neonatal period. Fetuses with diaphragmatic hernia were least likely to be terminated (24\%) but accounted for a quarter of the observed neonatal deaths relating to the nine anomalies. Just one in five fetuses with one of the nine selected anomalies were born alive and survived the neonatal period compared with four out of five for all other registered anomalies. Of fetuses with an anomaly, $84 \%$ (1159) were classified as "white," with the next largest ethnic groups being Pakistani (Asian or Asian British) (4\%, 53 fetuses), and Indian (Asian or Asian British) (3\%, 47 fetuses). Although birth data were not available by ethnic group, census data for the register area shows $2 \%$ of those aged $0-15$ were classified in the census as Pakistani and 3\% as Indian.

\section{Congenital anomalies in utero}

Poisson regression models of all anomalies combined showed a significant increase in the rate of registration of the selected anomalies over time, with rates 19\% higher in 2003-7 compared with 1998-2002 (rate ratio $1.19,95 \%$ confidence interval 1.08 to 1.32 ; table 2). There was no evidence of a difference in the overall rate of registrations by deprivation (rate ratio comparing the most deprived 10th with the least deprived 10th: 1.05, 0.90 to 1.23 ; table 3 ), although the rate ratio increased after adjustment for differences in maternal age (1.22, 1.04 to 1.44). When we looked at chromosomal and non-chromosomal anomalies separately, the effect of deprivation differed (table 3 ), with women from the most deprived 10th at increased risk of having a fetus with a non-chromosomal anomaly $(1.41,1.17$ to 1.70) but at reduced risk of a fetus with a chromosomal anomaly $(0.52,0.39$ to 0.69$)$ compared with those from the least deprived 10th. This latter effect was predominantly caused by the differences in maternal age between pregnant women from the most deprived and least deprived areas $(25 \%$ of fetuses were carried by mothers who were aged over 35 in the most deprived 10th compared with $64 \%$ in the least deprived 10th), and the increased risk of chromosomal anomalies with increasing maternal age (women over 35 were nearly five times more likely to carry a fetus with a chromosomal anomaly than younger mothers $(4.96,4.12$ to 5.98$))$. In contrast, there was no evidence of a difference in the rates of non-chromosomal anomalies with maternal age (1.01, 0.86 to 1.20$)$. The age adjusted rate ratio for chromosomal anomalies showed no evidence of a difference in rates of chromosomal anomalies with deprivation $(0.85,0.63$ to 1.15 )

\section{Antenatal detection}

In $86 \%$ of the affected fetuses the anomaly was detected during the antenatal period, and there was no evidence that this varied over time (rate ratio $1.09,0.98$ to 1.21 ), with deprivation ( 0.99 , 0.84 to 1.17$)$, or with maternal age $(0.95,0.84$ to 1.08$)$. A slightly lower proportion of cases from the most deprived areas was detected before 22 weeks' gestation compared with those from the least deprived areas, though this was not significant $(0.90,0.74$ to 1.08$)$. There was no evidence of a difference in antenatal detection rates by ethnicity $(\mathrm{P}=0.913)$. Antenatal detection by type of anomaly showed no evidence of a difference in antenatal detection rates with deprivation for either chromosomal $(0.96,0.70$ to 1.31$)$ or non-chromosomal anomalies (1.00, 0.82 to 1.23 ) (table 3 ).

\section{Terminations of pregnancy}

Rates of termination of pregnancy in which anomalies were detected antenatally were substantially lower in the most deprived 10th (63\%) compared with the least deprived 10th (79\%) (rate ratio $0.80,0.65$ to 0.97$)$, and this was similar after adjustment for differences in maternal age ( $0.79,0.65$ to 0.97$)$. There was no evidence of a change in the rate of termination over time $(\mathrm{P}=0.460)$ or with maternal age $(\mathrm{P}=0.968)$. The rate of termination was higher among those cases detected early (75\% at $<22$ weeks' gestation, $50 \%$ at $22-23$ weeks, and $19 \%$ at $\geq 24$ weeks), and adjustment for these differences slightly attenuated the effect of deprivation $(0.84,0.69$ to 1.03$)$. Rates of termination were similar for mothers classified as white British (71\%) or Indian (Asian or Asian British) $(71 \%)$ but considerably lower for Pakistani (Asian or Asian British) mothers $(42 \%)$. This led to a further small attenuation of the effect of deprivation $(0.86,0.70$ to 1.05$)$. The socioeconomic differences in rates of termination of pregnancy seen for all anomalies combined were similar to those seen for chromosomal and non-chromosomal anomalies considered separately (table $3)$.

\section{Fetal loss, stillbirth, live birth, and neonatal mortality associated with congenital anomalies}

Rates of live birth associated with an anomaly increased significantly over time by $27 \%$ from 1998-2002 to 2003-7. Socioeconomic variations in termination of pregnancy for congenital anomaly impacted greatly on socioeconomic inequalities in the rate of fetal loss, stillbirth, and live birth associated with an anomaly and also subsequent neonatal death. Considering all anomalies combined, compared with the least deprived 10th, the most deprived 10th had a $20 \%$ higher rate of stillbirth or fetal loss with an anomaly; a $61 \%$ higher rate of live 
birth with an anomaly; and a 98\% higher risk of neonatal mortality associated with an anomaly. After adjustment for differences in maternal age, socioeconomic inequality widened for women of a similar age: women in the most deprived 10th were $57 \%$ more likely to have a fetal loss or stillbirth, $85 \%$ more likely to have a liveborn baby with an anomaly, and $123 \%$ more likely to have a baby with an anomaly who died in the neonatal period.

Analysis by individual type of anomaly showed significant wide socioeconomic inequality for non-chromosomal anomalies. Compared with the least deprived 10th, rates in the most deprived 10th were $64 \%$ higher for live birth associated with a non-chromosomal anomaly and $130 \%$ higher for neonatal mortality associated with a non-chromosomal anomaly. In contrast, socioeconomic inequality for chromosomal anomalies seemed narrower and was not significant. In the most deprived 10th, rates of live birth of a baby with a chromosomal anomaly were $50 \%$ higher and rates of neonatal mortality were $44 \%$ higher compared with the least deprived 10th. Adjustment for the wide differences in maternal age, however, increased the socioeconomic inequality for chromosomal anomalies to $121 \%$ increased risk for a live birth of a baby with an anomaly and to $104 \%$ for a neonatal mortality associated with an anomaly. For chromosomal anomalies there was a reduced rate of fetal loss or stillbirth associated with an anomaly in the most deprived 10th compared with the least deprived 10th, but this was no longer evident after adjustment for maternal age and seemed to be related to the increased likelihood of the pregnancies of older mothers ending in a fetal loss or stillbirth.

\section{Sensitivity analyses}

Sensitivity analyses were undertaken to compare the postcode of residence at antenatal detection with postcode at delivery. Of the total 1579, this information was available for the 410 fetuses diagnosed antenatally in which the pregnancy did not end in termination. Thirty three mothers moved residence (8\%) between detection and delivery, and the median 10th of deprivation for these women was the same for postcode at antenatal detection and postcode at delivery.

We also carried out sensitivity analyses to compare the pattern of deprivation with outcome of pregnancy for all 13580 registered cases of anomaly. This analysis showed a similar pattern to the nine selected anomalies with a poor prognosis. There was an increase in the risk of the registration of an anomaly among women from the most deprived areas (rate ratio $1.25,1.19$ to 1.32 ) and a substantially lower rate of termination $(0.55,0.48$ to 0.62$)$, resulting in an increased rate of live birth $(1.48,1.39$ to 1.58$)$ and neonatal death $(1.98,1.49$ to 2.63$)$ associated with an anomaly.

\section{Discussion}

There are wide socioeconomic differentials in rates of termination of pregnancy for congenital anomalies that have a major impact on the observed socioeconomic inequalities in neonatal mortality. This has been sparsely reported previously because of a lack of routinely recorded data. While there is no evidence of a difference in overall rates of registered cases of the selected nine serious congenital anomalies "in utero," the socioeconomic variation in termination rates leads to a doubling of the rate of neonatal mortality associated with a serious congenital anomaly in the most deprived areas compared with the least deprived areas. Also, while socioeconomic inequalities in rates of anomaly in utero varied with type of anomaly, socioeconomic variations in termination of pregnancy led to a widening of the socioeconomic inequalities in the rate of live births and neonatal deaths associated with both chromosomal and non-chromosomal anomalies. We used data from the largest of the regional congenital anomaly registers in England. Although coverage of the UK population is incomplete, the data held by the nine regional registers are of the highest quality, and, since the demise of the National Congenital Anomaly System, are now the only available source of information for monitoring the incidence of congenital anomalies across the UK.

\section{Access to services}

A decision to continue a pregnancy associated with a serious congenital anomaly should not be thought of as a flawed choice and might relate to societal and cultural norms. It is important, however, that the reported socioeconomic variations in rates of termination do not arise from systematic differences in the delivery of services such as access to timely detection services, communication of risk of mortality and morbidity by health professionals, and access to termination of pregnancy. In terms of timely detection services, we found earlier detection of fetal anomalies was associated with higher rates of termination of pregnancy, confirming work by Rauch et al. ${ }^{18}$ While in England, Scotland, and Wales there is no gestational age limit at which a termination of pregnancy can be legally carried out for a serious fetal anomaly, if this is undertaken in the third trimester the fetus becomes potentially viable and hence termination at this stage is more likely to involve feticide and a process that involves a period of labour. In general, the ethical and psychological acceptability of termination of pregnancy for fetal anomaly is greater when it is performed earlier in pregnancy, suggesting that late booking limits a woman's options in terms of termination of pregnancy if a serious anomaly is detected.

We found no evidence of variation with socioeconomic classification or maternal age in the rates in antenatal detection of anomalies. Rates of early detection, however, were slightly lower in more deprived areas, leading to a slight attenuation of the socioeconomic variation in termination rates, suggesting that this could partially explain the variation. Antenatal care and screening for fetal anomaly need to be provided in an accessible way so that inequalities do not arise in the timing of when parents have to make such difficult decisions about what to do when faced with the news that their baby has a serious congenital anomaly. Furthermore, while we refer to "parents" making decisions, sole registrations of birth are higher among women from socioeconomically disadvantaged backgrounds. ${ }^{19}$

After detection of an anomaly, parents are referred to appropriate specialists and prenatal counsellors, which might involve additional investigations such as magnetic resonance imaging and karyotyping of the fetus and then communication of the risks of mortality and morbidity associated with the diagnosis. Socioeconomic differences might exist in health professionals' communication of these risks and how parents then interpret that risk. Finding out about a serious anomaly is an extremely stressful time, and the counselling needs to be unbiased and respectful of the parents' choice, culture, religion, and belief $\mathrm{s}^{20}$ for them to make an informed decision. Parents need to be allowed adequate time after counselling to reach their informed decision. Further research is needed to understand whether socioeconomic variation in rates of termination arises partially from socioeconomic differences in the communication and interpretation of risk and subsequent decision making. 


\section{Cultural and societal influences}

Cultural and societal factors concerning the acceptability of congenital anomalies and termination of pregnancy as a secondary prevention of anomalies are likely to affect the variations seen here. We did not have information on the extremely sensitive issue of women's decision making about whether to continue the pregnancy. We have shown here that women of Pakistani (Asian or Asian British) ethnicity have much lower rates of termination than white British or Indian (Asian or Asian British) women. We could not look at the important issues of religious beliefs and the acceptability of termination of pregnancy, but the ethnic differences seen here could partially reflect different attitudes to termination with religion. ${ }^{21}$ There is also discord in opinion within groups, ${ }^{22}$ and it is important that ethnicity or religion are not taken as a proxy for attitudes towards termination of pregnancy. Differences could be due to variations in the communication of risk and timing of detection as well as cultural or religious differences between ethnic groups. ${ }^{112324}$

Rates of termination of pregnancy did not vary by maternal age but varied widely by type of anomaly detected. These variations with anomaly probably reflect public understanding and acceptance of different types of anomalies and also the certainty of diagnostic information available to clinicians. The high termination rates for trisomy 13 , trisomy 18 , anencephaly, and spina bifida confirm previous research suggesting that women are more likely to choose a termination if the fetus is affected by chromosomal anomalies ${ }^{25}$ or anomalies of the central nervous system ${ }^{13}$ compared with other anomalies of similar severity. This could reflect a greater clinical certainty around these diagnoses or that parents believe chromosomal and central nervous system defects would compromise their child's quality of life more than other conditions. In contrast, in our study the rates of termination for diaphragmatic hernia were considerably lower and yet this anomaly accounted for the largest proportion of neonatal deaths. This is probably because of the relative uncertainty about prognosis and less public understanding of this condition. We found an increase in the registration of anomalies with a poor prognostic outcome over time. This is probably related partially to an improved rate of detection of these conditions and also to improved reporting over time throughout the region. We found no evidence of an interaction between time and deprivation, suggesting that this increase in detection is unlikely to vary by deprivation and hence should not affect the socioeconomic inequalities reported here.

\section{Limitations}

Alternative definitions of anomalies with a poor prognostic outcome could affect the results seen here. Our sensitivity analyses of all registered cases of congenital anomaly, however, showed similar findings to the nine selected anomalies. We focused on those anomalies with a high detection rate through the fetal anomaly screening programme. ${ }^{14}$ The socioeconomic inequalities in liveborn infants and neonatal deaths might be less marked for anomalies that are not routinely detected before birth as the differential rates of termination will not play a part. Other anomalies, such as Down's syndrome, rely on accessing additional blood tests and amniocentesis for detection and have much lower rates of associated mortality and morbidity. Such conditions might show greater socioeconomic inequalities in live births associated with an anomaly as there is greater potential for variation in access to screening and differences in interpretation of risk and, consequently, an increased likelihood of socioeconomic differences in decisions to terminate a pregnancy. Data from antenatal detection through to outcome of pregnancy are not available nationally in the UK or many other countries. We have therefore had to focus on a large population based register that covers $10 \%$ of England and Wales. As we are looking at 10 years of data this is a large population base of around 600000 births and provides detailed information from antenatal detection through to information on terminations, birth outcomes, and neonatal mortality unavailable elsewhere.

Our work lacks detailed data on individual deprivation measures and so could be open to problems of confounding. Despite this, provided the results are treated cautiously, our methods are relatively straightforward and provide a way for data from a congenital anomaly register to be used to monitor up to date trends in rates of antenatal detection, termination of pregnancy, prevalence of live birth, and mortality.

\section{Implications}

While this work concentrates on a specific region, we believe that these results are generalisable to the whole of the UK. The fetal anomaly screening programme aims to ensure consistent provision across the UK, although there might be some variation between centres in the antenatal detection rates of the selected anomalies and differences in uptake of screening programmes. We believe this is unlikely to impact substantially on socioeconomic differences in behaviour after detection of a suspected anomaly. In England, Scotland, and Wales, secondary prevention of anomalies through access to termination of pregnancy is available to all. Internationally this is not the case, but our findings could apply to countries with a similar policy on termination of pregnancy.

It is vital that variations in congenital anomalies arising through the secondary prevention schemes based on screening and the uptake of termination of pregnancy do not detract from the importance of reducing inequalities in anomalies through primary prevention before conception, as highlighted by Dolk. ${ }^{26}$ Further research into the links between non-chromosomal anomalies and deprivation needs to be undertaken to identify primary prevention interventions.

The use of prenatal diagnostic techniques and access to termination of pregnancy for fetal anomaly has reduced neonatal mortality internationally, ${ }^{6}$ but here we show that these secondary prevention measures have had a knock-on effect of an increase in the socioeconomic inequality in mortality. Future research into the reasons underlying the socioeconomic variations in continuation of pregnancies associated with serious congenital anomalies should assess whether this is because of systematic differences in access to services and communication and interpretation of risk.

We thank members of the NIHR programme grant team: Brad Manktelow, Elaine Boyle, and Julie Faulkes (University of Leicester) for helpful advice and comments throughout the study and Shona Golightly and Anna Springett (Centre for Maternal and Child Enquiries) for help with information on neonatal and infant deaths. We thank all NHS staff who notify cases to the EMSYCAR, Rosemary Pridding and Laura Berry for clerical support, and Martin Perkins for IT support.

Contributors: LKS undertook the statistical analysis and wrote the first draft of the paper. All authors made substantial contributions to conception, design, and interpretation of data and contributed to drafting the article or revising it critically for important intellectual content and approved the final manuscript. LKS, JLSB, and ESD were responsible for acquisition of data. LKS is the guarantor.

Funding: This study was funded by UK Department of Health's National Institute of Health Research (RM62G0225). EMSYCAR is funded by the primary care trust in the areas covered by the register. The sponsors 


\section{What is already known on this topic}

Socioeconomic inequalities exist in neonatal mortality

Congenital anomalies are a major cause of neonatal and infant mortality in developed countries

Screening for congenital anomaly and access to termination services have reduced neonatal mortality rates in many countries

\section{What this study adds}

Rates of antenatal detection of anomalies with a poor prognostic outcome are similar for all deprivation groups Rates of termination of pregnancy for congenital anomaly are lower in more deprived areas

Socioeconomic variation in rates of termination of pregnancy has led to a widening of socioeconomic inequalities in the rate of live birth associated with congenital anomaly and consequent neonatal mortality

had no input in the design, the conduct, the analysis, or the interpretation of the study, and did not influence the manuscript preparation.

Competing interests: All authors have completed the ICMJE uniform disclosure form at www.icmje.org/coi_disclosure.pdf (available on request from the corresponding author) and declare: no support from any organisation for the submitted work; no financial relationships with any organisations that might have an interest in the submitted work in the previous three years; no other relationships or activities that could appear to have influenced the submitted work.

Ethical approval: EMSYCAR is part of the British Isles Network of Congenital Anomaly Registers (BINOCAR), which have ethical approval from MREC (Trent MREC, date 11/09/2009 09/H0405/48) and NIGB (PIAG 2-08(e)/2002 20/06/2002) for studies involving the use of their data.

Data sharing: No additional data available.

1 Oakley L, Maconochie N, Doyle P, Dattani N, Moser K. Multivariate analysis of infant death in England and Wales in 2005-06, with focus on socio-economic status and deprivation. Health Stat Q 2009;42:22-39.

2 Smith LK, Manktelow BN, Draper ES, Springett A, Field DJ. An investigation of the nature of socioeconomic inequalities in neonatal mortality: a population based study. $B M J$ 2010;341:c6654

3 Neasham D, Dolk H, Vrijheid M, Jensen T, Best N. Stillbirth and neonatal mortality due to congenital anomalies: temporal trends and variation by small area deprivation scores in England and Wales, 1986-96. Paediatr Perinat Epidemiol 2001;15:364-73.

4 Olesen C, Thrane N, Ronholt AM, Olsen J, Henriksen TB. Association between social position and congenital anomalies: a population-based study among 19,874 Danish women. Scand J Public Health 2009;37:246-51.

5 Vrijheid M, Dolk H, Stone D, Abramsky L, Alberman E, Scott JE. Socioeconomic inequalities in risk of congenital anomaly. Arch Dis Child 2000;82:349-52.

6 Liu S, Joseph KS, Kramer MS, Allen AC, Sauve R, Rusen ID, et al. Relationship of prenatal diagnosis and pregnancy termination to overall infant mortality in Canada. JAMA 2002;287:1561-7.

7 Garne E, Loane M, Dolk H, De Vigan C, Scarano G, Tucker D, et al. Prenatal diagnosis of severe structural congenital malformations in Europe. Ultrasound Obstet Gynecol 2005;25:6-11.

8 Davidson N, Halliday J, Riley M, King J. Influence of prenatal diagnosis and pregnancy termination of fetuses with birth defects on the perinatal mortality rate in Victoria, Australia. Paediatr Perinat Epidemiol 2005;19:50-5.

9 Van der Pal-de Bruin KM, Graafmans W, Biermans MC, Richardus JH, Zijlstra AG, Reefhuis $\mathrm{J}$, et al. The influence of prenatal screening and termination of pregnancy on perinatal mortality rates. Prenat Diagn 2002;22:966-72.
10 Zeitlin J, Draper ES, Kollee L, Milligan D, Boerch K, Agostino R, et al. Differences in rates and short-term outcome of live births before 32 weeks of gestation in Europe in 2003: results from the MOSAIC cohort. Pediatrics 2008;121:e936-44.

11 Rowe RE, Garcia J, Davidson LL. Social and ethnic inequalities in the offer and uptake of prenatal screening and diagnosis in the UK: a systematic review. Public Health 2004;118:177-89.

12 Alderdice F, McNeill J, Rowe R, Martin D, Dornan J. Inequalities in the reported offer and uptake of antenatal screening. Public Health 2008;122:42-52.

13 Schechtman KB, Gray DL, Baty JD, Rothman SM. Decision-making for termination of pregnancies with fetal anomalies: analysis of 53,000 pregnancies. Obstet Gynecol 2002;99:216-22.

14 Kirwan D. NHS fetal anomaly screening programme: $18+0$ to $20+6$ weeks fetal anomaly scan national standards and guidance for England. NHS Fetal Anomaly Screening Programme, 2010.

15 Noble M, Wright G, Dibben C, Smith G, McLennan D, Anttila C, et al. Indices of deprivation 2004: report to the office of the deputy prime minister. Neighbourhood Renewal Unit, 2004.

16 Lumley T, Kronmal R, Ma S. Relative risk regression in medical research: models, contrasts, estimators, and algorithms. UW Biostatistics Working Paper Series, 2006. 7 Oehlert GW. A note on the delta method. Am Stat 1992;46:27-9.

18 Rauch ER, Smulian JC, DePrince K, Ananth CV, Marcella SW. Pregnancy interruption after second trimester diagnosis of fetal structural anomalies: the New Jersey Fetal Abnormalities Registry. Am J Obstet Gynecol 2005;193:1492-7.

19 Smallwood S. Characteristics of sole registered births and the mothers who register them. Popul Trends 2004;20:6

20 Gagnon A, Wilson RD, Allen VM, Audibert F, Blight C, Brock JA, et al. Evaluation of prenatally diagnosed structural congenital anomalies. J Obstet Gynaecol Can 2009;31:875-89.

21 Hewison J, Green JM, Ahmed S, Cuckle HS, Hirst J, Hucknall C, et al. Attitudes to prenata testing and termination of pregnancy for fetal abnormality: a comparison of white and Pakistani women in the UK. Prenat Diagn 2007;27:419-30.

22 Arif MO, Fatmi Z, Pardeep B, Ali T, lqbal H, Bangash HK, et al. Attitudes and perceptions about prenatal diagnosis and induced abortion among adults of Pakistani population. Prenat Diagn 2008;28:1149-55.

23 Ahmed S, Hewison J, Green JM, Cuckle HS, Hirst J, Thornton JG. Decisions about testing and termination of pregnancy for different fetal conditions: a qualitative study of European White and Pakistani mothers of affected children. J Genet Couns 2008;17:560-72.

24 Ahmed S, Green JM, Hewison J. Attitudes towards prenatal diagnosis and termination of pregnancy for thalassaemia in pregnant Pakistani women in the North of England. Prenat Diagn 2006;26:248-57.

25 Zyblewski SC, Hill EG, Shirali G, Atz A, Forbus G, Gonzalez J, et al. Chromosomal anomalies influence parental treatment decisions in relation to prenatally diagnosed congenital heart disease. Pediatr Cardiol 2009;30:1105-11.

26 Dolk H. What is the "primary" prevention of congenital anomalies? Lancet 2009;374:378.

Accepted: 16 May 2011

Cite this as: BMJ 2011:343:d4306 


\section{Tables}

Table 1| Number of registered cases, antenatal detection rate, and outcome of pregnancy by anomaly 1998-2007. Figures are percentage (number) except for total cases

\begin{tabular}{|c|c|c|c|c|c|c|c|c|}
\hline & \multirow[b]{2}{*}{ ICD code } & \multirow[b]{2}{*}{$\begin{array}{c}\text { Total No of } \\
\text { cases }\end{array}$} & \multirow[b]{2}{*}{$\begin{array}{l}\text { Antenatal } \\
\text { detection }\end{array}$} & \multirow[b]{2}{*}{$\begin{array}{l}\text { Termination in } \\
\text { cases detected } \\
\text { antenatally }\end{array}$} & \multicolumn{4}{|c|}{ Outcome of pregnancy for all cases } \\
\hline & & & & & Termination & $\begin{array}{l}\text { Fetal loss and } \\
\text { stillbirth }\end{array}$ & $\begin{array}{c}\text { Live birth } \\
\text { (surviving }>28 \\
\text { days) }\end{array}$ & Neonatal death \\
\hline \multicolumn{9}{|l|}{ Selected anomalies: } \\
\hline Anencephaly & Q000 & 257 & $97(249)$ & $88(218)$ & $85(218)$ & $8(20)$ & $0(1)$ & $7(18)$ \\
\hline Spina bifida & Q050-Q059 & 339 & $90(303)$ & $78(235)$ & $70(235)$ & $6(20)$ & $22(75)$ & $2(8)$ \\
\hline Hypoplastic left heart & Q234 & 171 & $85(146)$ & $56(82)$ & $48(82)$ & $8(14)$ & $25(42)$ & $19(33)$ \\
\hline $\begin{array}{l}\text { Bilateral renal } \\
\text { agenesis }\end{array}$ & Q601/606 & 59 & $81(48)$ & $85(41)$ & $69(41)$ & $15(9)$ & $0(0)$ & $15(9)$ \\
\hline $\begin{array}{l}\text { Lethal skeletal } \\
\text { dysplasia }\end{array}$ & Q771-3 & 45 & $82(37)$ & $57(21)$ & $47(21)$ & $7(3)$ & $33(15)$ & $13(6)$ \\
\hline Diaphragmatic hernia & Q790 & 183 & $77(140)$ & $29(40)$ & $22(40)$ & $8(14)$ & $47(86)$ & $23(43)$ \\
\hline Exomphalos & Q792 & 230 & $85(195)$ & $56(109)$ & $47(109)$ & $15(34)$ & $34(77)$ & $4(10)$ \\
\hline Trisomy 18 & Q910-13 & 285 & $85(242)$ & $81(196)$ & $69(196)$ & $13(38)$ & $6(18)$ & $12(33)$ \\
\hline Trisomy 13 & Q914-7 & 127 & $84(107)$ & $83(89)$ & $70(89)$ & $16(20)$ & $5(6)$ & $9(12)$ \\
\hline \multicolumn{9}{|c|}{ Other anomalies audited by fetal anomaly screening programme: } \\
\hline Cleft lip & Q360-Q379 & 540 & - & - & $6(33)$ & $3(17)$ & $90(484)$ & $1(6)$ \\
\hline Gastroschisis & Q793 & 278 & - & - & $6(17)$ & $6(16)$ & $87(242)$ & $1(3)$ \\
\hline All selected anomalies & - & 1579 & $86(1357)$ & $70(944)$ & $60(944)$ & $10(158)$ & $20(317)$ & $10(160)$ \\
\hline Other registered & - & 12001 & 一 & 一 & $13(1514)$ & $5(550)$ & $80(9579)$ & $3(358)$ \\
\hline Total registered & - & 13580 & - & - & $18(2458)$ & $5(708)$ & 73 (9896) & $4(518)$ \\
\hline
\end{tabular}


Table 2| Rate of pregnancies associated with anomaly, rate of antenatal detection, and outcome of pregnancy by deprivation and year of birth based on Poisson regression model: all anomalies combined

\begin{tabular}{|c|c|c|c|c|c|}
\hline \multirow[b]{2}{*}{ All selected anomalies $(n=1579)$} & \multicolumn{2}{|c|}{ Least deprived 10th } & \multicolumn{2}{|c|}{ Most deprived 10th } & \multirow{2}{*}{$\begin{array}{l}\text { Change over time adjusted for } \\
\text { deprivation 2003-7 } v \text { 1998-2002 }\end{array}$} \\
\hline & 1998-2002 & 2003-7 & 1998-2002 & 2003-7 & \\
\hline Registered cases/10 000 births & 24.0 (21.4 to 26.8$)$ & 28.6 (25.7 to 31.9$)$ & 25.2 (22.7 to 27.9 ) & 30.1 (27.3 to 33.1$)$ & 1.19 (1.08 to 1.32$)$ \\
\hline$\%$ of all cases detected antenatally & $82.3(72.8$ to 93.1$)$ & 89.4 (79.6 to 1.00$)$ & $81.7(73.1$ to 91.4$)$ & 88.8 (80.1 to 98.3$)$ & $1.09(0.98$ to 1.21$)$ \\
\hline $\begin{array}{l}\% \text { terminations in cases detected } \\
\text { antenatally }\end{array}$ & 80.7 (70.0 to 93.0$)$ & 76.9 (67.1 to 88.2$)$ & $64.4(56.2$ to 73.9$)$ & $61.4(54.1$ to 69.6$)$ & 0.95 (0.84 to 1.08$)$ \\
\hline Stillbirth or late fetal loss/10 000 births & 2.7 (1.9 to 3.8$)$ & $2.2(1.5$ to 3.2$)$ & $3.2(2.4$ to 4.4$)$ & 2.7 (2.0 to 3.7$)$ & $0.82(0.60$ to 1.13$)$ \\
\hline Live birth per 10 000/live births & 5.5 (4.4 to 6.9$)$ & $7.0(5.7$ to 8.6$)$ & $8.9(7.4$ to 10.7$)$ & 12.3 (11.3 to 13.3$)$ & 1.27 (1.06 to 1.52$)$ \\
\hline Neonatal deaths/10 000 live births & $1.8(1.2$ to 2.6$)$ & 1.9 (1.3 to 2.8$)$ & 3.5 (2.6 to 4.8$)$ & 3.8 (2.9 to 5.1$)$ & $1.08(0.79$ to 1.47$)$ \\
\hline
\end{tabular}


Table 3| Rate of pregnancies associated with anomaly, rate of antenatal detection, and outcome of pregnancy by deprivation adjusted for year of birth based on Poisson regression model for all anomalies combined and by type of anomaly. Figures are rate ratios for deprivation gap (most deprived 10th $v$ least deprived 10th), unadjusted or adjusted for maternal age

\begin{tabular}{|c|c|c|c|c|c|c|}
\hline & \multicolumn{2}{|c|}{ All selected anomalies $(n=1579)$} & \multicolumn{2}{|c|}{ Non-chromosomal anomalies $(n=1118)$} & \multicolumn{2}{|c|}{ Chromosomal anomalies $(n=461)$} \\
\hline & Unadjusted & Adjusted & Unadjusted & Adjusted & Unadjusted & Adjusted \\
\hline Registered cases/10 000 births & $1.05(0.90$ to 1.23$)$ & $1.22(1.04$ to 1.44$)$ & $1.41(1.17$ to 1.70$)$ & $1.43(1.17$ to 1.74$)$ & $0.52(0.39$ to 0.69$)$ & $0.85(0.63$ to 1.15$)$ \\
\hline $\begin{array}{l}\% \text { of all cases detected } \\
\text { antenatally }\end{array}$ & 0.99 (0.84 to 1.17$)$ & 0.99 (0.84 to 1.18$)$ & 1.00 (0.82 to 1.23$)$ & $1.01(0.82$ to 1.24$)$ & $0.96(0.70$ to 1.31$)$ & $0.97(0.70$ to 1.35$)$ \\
\hline $\begin{array}{l}\text { \% terminations in cases detected } \\
\text { antenatally }\end{array}$ & $0.80(0.65$ to 0.98$)$ & 0.79 (0.64 to 0.98$)$ & 0.84 (0.66 to 1.08$)$ & $0.82(0.64$ to 1.06$)$ & $0.82(0.57$ to 1.17$)$ & $0.82(0.56$ to 1.19$)$ \\
\hline Stillbirth or fetal loss/10 000 births & $1.20(0.74$ to 1.97$)$ & 1.57 (0.93 to 2.63$)$ & 2.47 (1.26 to 4.86$)$ & 2.57 (1.26 to 5.24$)$ & $0.47(0.22$ to 1.00$)$ & 0.85 (0.38 to 1.86$)$ \\
\hline Live birth/10 000 live births & 1.61 (1.21 to 2.15$)$ & 1.85 (1.36 to 2.50$)$ & $1.64(1.20$ to 2.24$)$ & 1.78 (1.28 to 2.48$)$ & $1.50(0.74$ to 3.04$)$ & $2.21(1.05$ to 4.64$)$ \\
\hline Neonatal deaths/10 000 live births & $1.98(1.20$ to 3.27$)$ & $2.23(1.31$ to 3.78$)$ & 2.30 (1.25 to 4.23$)$ & 2.32 (1.22 to 4.42$)$ & $1.44(0.60$ to 3.47$)$ & $2.04(0.81$ to 5.14$)$ \\
\hline
\end{tabular}

